\title{
BMJ Open Development and usability testing of HEARTPA + N: protocol for a mixed methods strategy to develop an integrated smartphone and web-based intervention for women with cardiac pain
}

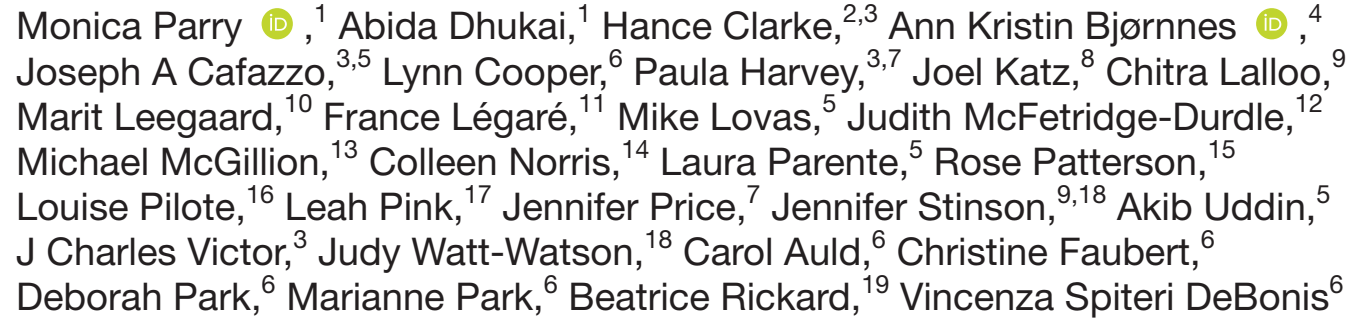

\section{ABSTRACT}

Introduction More women experience cardiac pain related to coronary artery disease and cardiac procedures compared with men. The overall goal of this programme of research is to develop an integrated smartphone and webbased intervention (HEARTPA $\mathrm{N}$ ) to help women recognise and self-manage cardiac pain.

Methods and analysis This protocol outlines the mixed methods strategy used for the development of the HEARTPA $O N$ content/core feature set (phase 2A), usability testing (phase 2B) and evaluation with a pilot randomised controlled trial (RCT) (phase 3). We are using the individual and family self-management theory, mobile device functionality and pervasive information architecture of mHealth interventions, and following a sequential phased approach recommended by the Medical Research Council to develop HEARTPA $\odot N$. The phase 3 pilot RCT will enable us to refine the prototype, inform the methodology and calculate the sample size for a larger multisite RCT (phase 4, future work). Patient partners have been actively involved in setting the HEARTPA $\bigcirc \mathrm{N}$ research agenda, including defining patient-reported outcome measures for the pilot RCT: pain and health-related quality of life (HRQoL). As such, the guidelines for Inclusion of Patient-Reported Outcomes in Clinical Trial Protocols (SPIRIT-PRO) are used to report the protocol for the pilot RCT (phase 3). Quantitative data (eg, demographic and clinical information) will be summarised using descriptive statistics (phases $2 A B$ and 3 ) and a content analysis will be used to identify themes (phase $2 A B$ ). A process evaluation will be used to assess the feasibility of the implementation of the intervention and a preliminary efficacy evaluation will be undertaken focusing on the outcomes of pain and HRQoL (phase 3).

\section{Strengths and limitations of this study}

- Robust methods guided by the individual and family self-management theory, mobile device functionality and the sequential phased approach recommended by the Medical Research Council.

- Sustainable HEARTPA ${ }_{+} \mathrm{N}$ design and development based on the real needs of women with oversight by a Patient Advisory Committee.

- Extensive recruitment and solid retention strategies using gender and culturally sensitive research methods.

- Larger pilot randomised controlled trial (RCT) focused on feasibility and primary evaluation of efficacy will increase precision of estimates and provide robust data to inform the design of a future full-scale RCT.

Ethics and dissemination Ethics approval was obtained from the University of Toronto (36415; 26 November 2018). We will disseminate knowledge of HEARTPA $P \mathrm{~N}$ through publication, conference presentation and national public forums (Café Scientifique), and through fact sheets, tweets and webinars.

Trial registration number NCT03800082.

\section{INTRODUCTION}

Cardiac pain is a key symptom of coronary artery disease (CAD) and acute coronary syndrome. Women have a varied pattern and distribution of cardiac pain and/or cardiac pain symptoms associated with both obstructive (macrovascular) and non-obstructive 
(microvascular) CAD. Women with obstructive CAD are usually 7-10 years older than men ${ }^{1}$ and present with coronary atherosclerosis and risk of atherosclerotic plaque rupture and/or erosion. ${ }^{2}$ Compared with men, women with obstructive $\mathrm{CAD}$ who undergo a percutaneous coronary intervention (PCI) ${ }^{3}$ and/or cardiac surgery ${ }^{4-6}$ have more persistent pain of moderate to severe intensity. ${ }^{7}$ The origin of this pain is complex, and thought to be pathophysiologic (eg, scar tissue, damage to intercostal nerves) and/or psychological (eg, anxiety) in origin. ${ }^{8}$ Non-obstructive CAD is cardiac pain without evidence of coronary artery obstruction, ${ }^{9}$ defined as less than a $50 \%$ epicardial coronary lesion on angiography. ${ }^{10}$ Coronary microvascular dysfunction/coronary spasm and coronary microembolism also contribute to ischaemia in non-obstructive CAD. ${ }^{11}$ Recent evidence suggests that up to $67 \%$ of women who present with cardiac pain and/ or cardiac pain symptoms have ischaemia related to non-obstructive CAD. ${ }^{12}$ Non-obstructive CAD is more prevalent in younger, middle-aged women and evidence suggests that more extensive, non-obstructive CAD is associated with major adverse cardiac events similar to those with obstructive $\mathrm{CAD} .{ }^{13}$ Obstructive/non-obstructive CAD is the leading cause of death of women across all ages, and recent data show an increase in $\mathrm{CAD}$ incidence and deaths among women $45-54$ years of age. ${ }^{14}$

Many women describe typical obstructive and nonobstructive cardiac pain as tight, heavy and dull with additional symptoms that include nausea and palpitations, ${ }^{15}$ and/or dyspnoea, weakness and unusual fatigue. ${ }^{16}$ Women also report that their cardiac pain is more likely to radiate to their left arm, back and/or jaw and neck. ${ }^{15}$ Women describe persistent poststernotomy pain as aching, tender and exhausting. ${ }^{4}$ This varied pattern and distribution of symptoms make it difficult for women to interpret as cardiac related (ie, obstructive/non-obstructive or post-PCI/cardiac surgery). ${ }^{13} 1718$ Women also minimise symptoms, prefer to consult with family and friends and have caring responsibilities and concerns for their family. ${ }^{19}$ As a result, women delay seeking appropriate care for their cardiac pain. ${ }^{20}$ The time from symptom onset to emergency department (ED) arrival for women is $85-320 \mathrm{~min}$, this has not changed in the last decade. ${ }^{21}$

Women with cardiac pain due to obstructive/nonobstructive $\mathrm{CAD}$ and/or post-PCI/cardiac surgery pain are frequent users of healthcare services ${ }^{22}$ and at risk for impaired function, depression, poor health-related quality of life (HRQoL) and death. ${ }^{23}$ Women have been historically under-represented in cardiovascular clinical trials ${ }^{24}{ }^{25}$ with much of the current evidence comparing cardiac pain and/or cardiac pain symptoms in women to men. There is little evidence focused on interventions to assist women to recognise and manage cardiac pain and/or cardiac pain symptoms ${ }^{26}$ Self-management interventions allow people to take an active part in the management of their own conditions ${ }^{27}$ and are important predictors of successful behaviour change. ${ }^{28}$ In addition to reducing pain, self-management interventions improve
HRQoL. ${ }^{29-34}$ A current mixed methods systematic review of self-management programmes (HEARTPA $+\mathrm{N}$, phase 1), which included women greater than 18 years of age with cardiac pain, found self-management interventions for cardiac pain were more effective if they included a greater proportion of women $(\mathrm{p}=0.02)$, goal setting $(p=0.03)$ and collaboration/support from healthcare providers (HCP) $(p=0.01) .{ }^{35}$ Mobile health (mHealth) technologies have been developed to help women selfmanage weight, ${ }^{36-40}$ increase physical activity, ${ }^{41}$ monitor for perinatal depression and assist with postpartum smoking cessation. ${ }^{42}$ Many women view mobile health technologies as novel and supportive, ${ }^{36}$ and indicate these technologies motivate healthy behaviours, reduce symptoms ${ }^{43}$ and improve HRQoL. ${ }^{43}$ Health app usage across all ages is on the rise, ${ }^{445}$ yet there is little objective rigorous research evaluating outcomes of smartphonebased interventions. ${ }^{46}$ The benefits of mHealth interventions in healthcare are compelling; smartphones are portable, they offer connectivity and they provide access to women who are difficult to reach, yet no smartphone or web-based self-management programme has been developed and tested with women who have cardiac pain and/or cardiac pain symptoms.

\section{OBJECTIVES}

The overall goal of this programme of research is to develop and systematically evaluate an integrated smartphone and web-based intervention (HEARTPA $+\mathrm{N}$ ) to provide evidence-informed symptom triage and self-management support to reduce pain and increase HRQoL in women with cardiac pain and/or cardiac pain symptoms. Specific objectives for each phase of development/evaluation include: (1) develop the HEARTPA우 $\mathrm{N}$ content and core feature set (phase 2A), (2) conduct usability testing (phase $2 B$ ), and (3) assess feasibility in terms of implementation (accrual rates, acceptability and level of engagement) and determine an initial estimation of effectiveness outcomes (estimates of magnitude of effect) in a pilot randomised controlled trial (RCT) (phase 3). The phase 3 pilot study will enable us to refine the prototype, inform the methodology and calculate the sample size for a larger multisite RCT (phase 4, future work).

\section{METHODS AND ANALYSIS \\ Phases 2A and 2B}

We are using the individual and family self-management theory, ${ }^{47} 48$ mobile device functionality and the pervasive information architecture of mHealth interventions, ${ }^{49}$ and following the sequential phased approach recommended by the Medical Research Council (MRC) ${ }^{50-52}$ and used by Stinson and others ${ }^{5253}$ to develop HEARTPAN. We will develop the HEARTPA $P \mathrm{~N}$ content/core feature set and conduct usability testing (phases $2 A$ and $2 B$ ) to ensure it is easy to use, efficient and satisfying to operate. 
Study design

Focus group interviews in phase $2 A$ will assist to: (1) learn about the experiences and healthcare needs of women with cardiac pain/cardiac pain symptoms from the perspectives of women, (2) design content and the core feature set of HEARTPA $9 \mathrm{~N}$, and (3) validate the HEART$\mathrm{PA}+\mathrm{N}$ triage algorithms with HCPs. This feature set will include evidence-informed symptom triage algorithms to help women recognise their cardiac pain/cardiac pain symptoms and seek appropriate care. The usability testing in phase $2 B$ will focus on user performance (ease of use, efficiency, ease of learning and errors) and satisfaction with programme content and functionality (reports, goal setting). ${ }^{54}$

\section{Eligibility criteria}

Women living in Canada greater than 18 years of age with obstructive/non-obstructive $\mathrm{CAD}$ pain and/or pain post-PCI/cardiac surgery lasting greater than 3 months. All women will be required to speak and read English and will be excluded if they have severe cognitive impairment assessed using the Six-Item Screener administered by telephone or in face-to-face interview, ${ }^{55} 56$ or major comorbid medical or psychiatric illness that could preclude their ability to participate in an interview. HCPs will include physicians and nurses/nurse practitioners who have worked in cardiology, family medicine or in an ED for at least 1 year; trainees, whose presence in the clinical setting is often transient, will be excluded.

\section{Study setting}

Phase 2A 1-hour focus group interviews will be scheduled at a mutually convenient time for participants, and conducted by telephone, using ZOOM online video conferencing technology, or face to face in a location suitable to participants and free from distractions. ${ }^{57}$ Phase $2 B$ participants will complete a one-on-one observation for 60-90 min in a quiet room within the labs at Healthcare Human Factors in Toronto, Ontario, Canada.

\section{Procedures}

Phase 2A. Following ethics approval, a purposive sample of women with obstructive $(n=10) /$ non-obstructive $(n=10)$ CAD pain and post-PCI/cardiac surgery pain $(n=10)$ will be recruited for focus group interviews through cardiology, cardiac surgery and pain clinics, and using social media platforms (eg, Twitter, Facebook). HCPs $(n=10)$ will also be recruited for a separate focus group interview via letters and emails. We will use a semistructured interview guide to explore the views, experiences and beliefs/ motivations $^{57}$ of women with cardiac pain. We will also use a semistructured interview guide to validate the triage algorithms with HCPs. Interviews will be conducted by two team members experienced in conducting interviews and techniques will be used to minimise power differentials, such as establishing rapport, active listening and relaxed body language. ${ }^{58}$
Phase 2B. Based on previous experience $\mathrm{e}^{59-61}$ and recommendations that usability testing by three to five users finds approximately $85 \%$ of interface usability problems, ${ }^{62} 63$ each usability cycle will include five end users (per pain type-obstructive $(n=5) /$ non-obstructive $(n=5)$ CAD, and pain post-PCI/cardiac surgery $(n=5))$. Women will be provided with a brief explanation of the HEART$\mathrm{PA}+\mathrm{N}$ intervention and then asked to move through standardised scenarios and list of features including the about you, event profile, goal-setting features, graphics, audio and video clips and interactive components (reporting, symptom triage algorithms, self-management skills). We will employ a 'think aloud' approach ${ }^{64}$ to gather insight into the way users solve problems as they move through the application in a systematic way. Comments will be recorded, and the project coordinator will make field notes about any problems encountered on the Usability Testing Error and Efficiency Documentation Form. At the end of the session, participants will be asked to complete the System Usability Scale (SUS). ${ }^{65}$ The SUS has been used across a wide range of user interfaces, including web pages and web applications. ${ }^{66}$ The ten 5 -point Likert questions can be scored to provide a point estimate of usability with a reported reliability of $0.85 .{ }^{67}$ In addition, four semistructured questions will be asked to determine users' overall impression of $\mathrm{HEARTPA}_{+} \mathrm{N}$, what they liked and why, what could be improved and if anything was missing. ${ }^{61}$ Observations will be conducted in iterative cycles. After the first cycle, changes will be made to the interface based on comments from the content analysis of the audiotapes and field notes. The revised user interface will then be evaluated in a subsequent cycle. These iterations usually require two to three testing cycles with each end user group until no further comments are identified. $^{616267}$

\section{Outcomes}

We will use the summary matrix from our integrated mixed methods systematic review (phase 1$)^{35}$ and the results from focus group interviews (phase 2A) to discuss HEARTPA $+\mathrm{N}$ designs with women who have cardiac pain in a consensus workshop with our Human Factors Designers. HEARTPA $+\mathrm{N}$ will be designed for a consistent experience for women, and developed on a webbased platform, with easy access on any device with a web browser, including smartphones and tablets. ${ }^{68} 69$ HEARTPAN's web-based approach will allow for faster maintenance, easier updates to content, as well as improved accessibility for users. ${ }^{70}$ All HEARTPA $9 \mathrm{~N}$ contents will be written at a grade 5-6 reading level, ${ }^{71}$ and communication with a central database server will occur through secure internet connections. Women with cardiac pain will be able to participate in realistic scenarios in a simulated environment (phase 2B) in order to assess the appropriateness and ease of use of HEARTPA $+\mathrm{N}$ prior to the phase 3 pilot RCT. 


\section{Phase 3}

The HEARTPA $q \mathrm{~N}$ intervention is the first of its kind; there are no previous trials of the efficacy of such an intervention to decrease pain and improve HRQoL in women with cardiac pain/cardiac pain symptoms. We will undertake a process and preliminary effect evaluation of the HEARTPA $+\mathrm{N}$ intervention for women with cardiac pain, as guided by the MRC framework. ${ }^{50-52}$ The guidelines for Inclusion of Patient-Reported Outcomes in Clinical Trial Protocols (SPIRIT-PRO) are used to report the protocol for this pilot RCT.

\section{Study design}

A two-group parallel single-blind pilot RCT.

\section{Eligibility criteria}

Inclusion/exclusion criteria have been previously described (phases $2 A$ and 2B). Additional exclusion criteria will include women who participated in phase $2 \mathrm{~A}$ or phase $2 B$ studies.

\section{Study setting}

Participants will attend one in-person session to learn about the trial, obtain informed written consent and complete demographic, clinical and baseline measures (T1). Participants allocated to the intervention group will also learn how to use the HEARTPA $9 \mathrm{~N}$ intervention. The intervention will be delivered on restricted passwordprotected applications.

\section{Procedures}

Following ethics approval, a single coordinating centre (University of Toronto) will recruit women using the methods described previously (phases $2 A$ and $2 B$ ). Interested participants will contact the project coordinator by telephone or express their interest using the HEART$\mathrm{PA} \not \mathrm{N}$ website. Eligibility criteria will be confirmed, verbal consent obtained and an appointment for an initial study visit will be made. The project coordinator will track the number of eligible participants approached and reasons for refusal using a study log. We will use multiple methods to promote recruitment and retention, such as reimbursing participants for travel costs related to the initial study visit and reimbursement for use of their smartphone and data plan (\$85) for the duration of the study. The project coordinator will send email and postcard reminders and at 3 months, participants will be telephoned (standardised script) to complete post-test measures online at home. Gift cards will be provided at study completion $(\$ 25)$. We anticipate minimal loss to follow-up as reported in previous pilot studies. ${ }^{72}$ However, logins every 1-2 days for 3 months may be burdensome for women, which we will assess in our process evaluation. The project coordinator will also be available to address questions, issues and concerns without delay and all T2 assessments will be completed online, eliminating the need for participants to return to the study centre.

Randomisation. Following completion of baseline measures, participants will be randomised to the control or intervention group at a 1:1 ratio in blocks of 4 stratified by type of cardiac pain ${ }^{73} 74$ (obstructive CAD, non-obstructive CAD and post-PCI/cardiac surgery). Randomisation will be managed centrally using a webbased randomisation service (www.randomize.net/).

Allocation. Participants allocated to the control group will receive the usual care and supports provided to women with cardiac pain/cardiac pain symptoms, including usual clinic appointments and follow-up. With detailed informed consent procedures, it is expected that women will accept their group allocation following randomisation. Participants randomised to the intervention group will consist of use of the HEARTPA $+\mathrm{N}$ intervention every 1-2 days, in addition to usual care, for a period of 3 months. The HEARTPA $9 \mathrm{~N}$ intervention will be delivered on restricted password-protected applications that will permit tracking of adherence (number of logins to app and website using Google Analytics). Participants will be encouraged to log in to HEARTPA $9 \mathrm{~N}$ every 1-2 days (via automated alerts) over the 3-month period to develop and track goals related to pain, activities, sleep and emotions. Participants will be directed to the project coordinator for technical problems.

Blinding. It is not possible to blind the participants to group allocation due to the specific nature of the HEARTPA $9 \mathrm{~N}$ intervention; however, a data analyst at the University of Toronto's Faculty of Nursing who is blinded to treatment allocation will conduct the analysis ensuring neutrality of the outcome assessment.

\section{Outcomes}

A process evaluation will be used to assess the feasibility of the implementation of the intervention. Recruitment and retention will be determined through the use of the study log, which will document each potential participant contacted, whether or not they chose to participate in the trial, reasons for non-participation, whether or not they completed follow-up assessments and reasons for dropout. Issues and/or difficulties encountered during trial implementation will be tracked. Adverse events will be recorded on an Adverse Event Form and engagement will be assessed using Google Analytics. We will assess acceptability and satisfaction at the end of the 3-month period in all participants in the intervention group using a modified Acceptability e-Scale (AES). ${ }^{75}$ A preliminary efficacy evaluation will also be undertaken focusing on the outcomes of pain and HRQoL. Pain will be measured using the Brief Pain Inventory-Short Form (BPI-SF), which rates pain severity and the degree to which pain interferes with mood, sleep and other physical activities such as work, social activity and relations with others. It has good construct validity, ${ }^{76}$ reliability is reported at $0.86-0.91^{76}$ and it has detected clinically important differences. ${ }^{4278}$ HRQoL will be measured using the SF-36v2, which contains 36 items and yields a score for each of the eight domains of health: physical functioning, role limitations due to physical health (role-physical), bodily pain, general health perceptions, vitality, social 


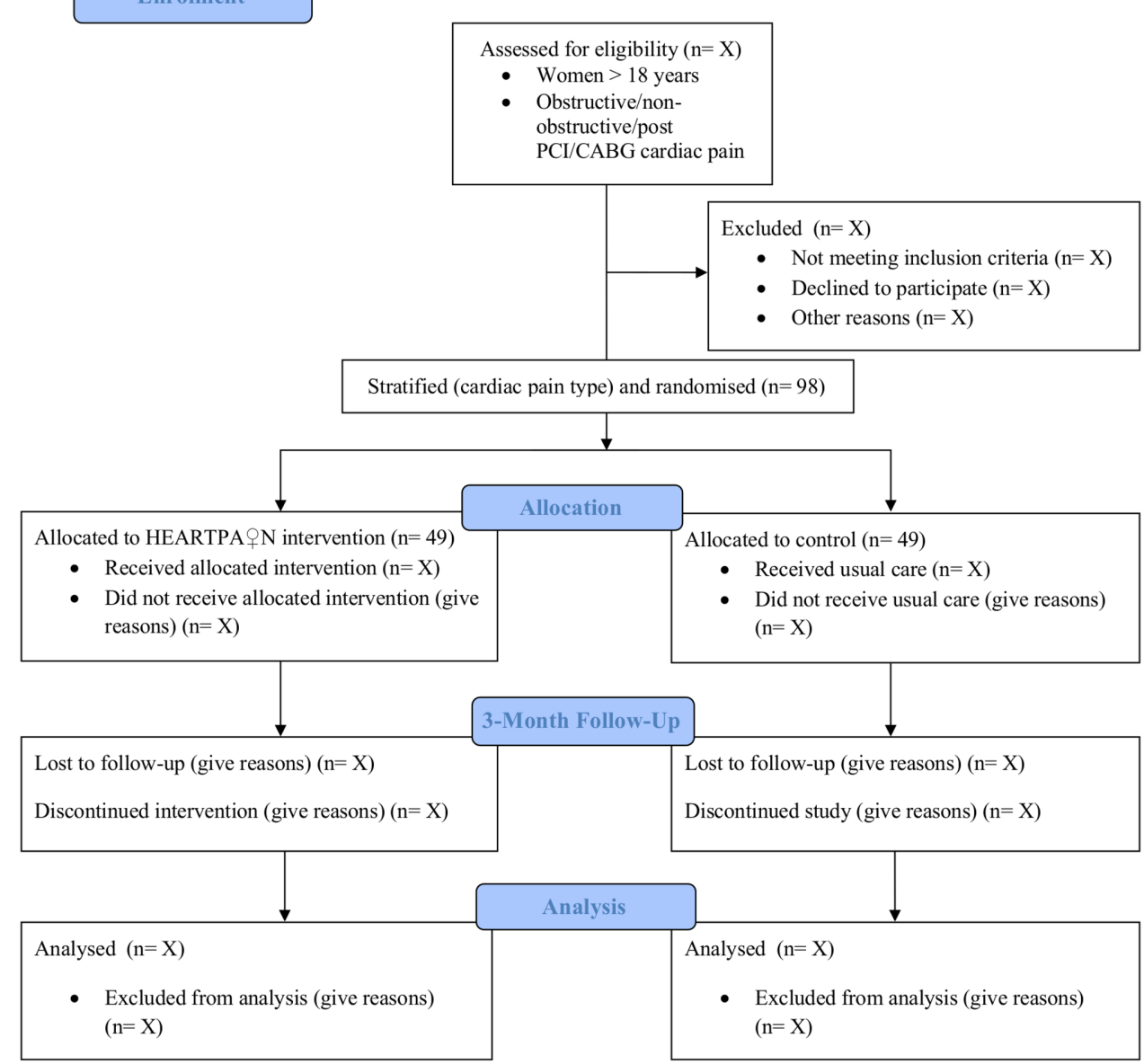

Figure 1 Anticipated participant flow-through in pilot randomised controlled trial (RCT). CABG, coronary artery bypass graft; $\mathrm{PCl}$, percutaneous coronary intervention.

functioning, role limitations due to emotional problems (role-emotional) and mental health. ${ }^{79}$ It has an internal consistency of $0.76-0.94^{80} 81$ with construct, criterion and predictive validity. ${ }^{81}$ A participant flow diagram is included in figure 1.

\section{Sample size}

As this is a pilot trial focused on feasibility and primary evaluation of efficacy, we are not testing for statistical significance. ${ }^{82}$ To decide on a sample size, we used the CI approach based on the feasibility outcomes of recruitment and retention. For a one-sided $95 \%$ CI for the proportion of women recruited and a margin of error of 0.05 (the lower bound) we would need at least 81 participants to estimate an overall recruitment rate of 0.70 . A sample of $\mathrm{n}=49$ would be needed to estimate an overall retention rate of $85 \%$; however, to estimate retention separately in the intervention and control groups, we will need a total sample size of $98(49 * 2)$. As attrition is one of our measured feasibility outcomes, we have not accounted for it in the sample size calculations.

\section{Data management}

Data will be collected using the HEARTPA $9 \mathrm{~N}$ application, as well as surveys and stored on a password-protected server. The trial steering committee includes all research and Patient Advisory Committee (PAC) team members. As this is a pilot trial, there is not a separate data monitoring and safety committee.

\section{Statistical methods}

Process evaluation. Prevalence of refusal, retention, engagement with the intervention and technical difficulties reported will be calculated, along with their $95 \%$ CIs. Mean acceptability and satisfaction will be calculated from the total score of the AES, along with its SD. We will record symptom descriptions and use of the symptom triage algorithms, what women did as a result of this recommendation (eg, self-management, contact with primary HCP, ED visit). Qualitative process data collected will be analysed using methods appropriate to the data obtained.

Primary effect evaluation. We will investigate the variability and sensitivity to change for outcomes of pain and HRQoL (T2-T1). We will calculate the number of participants who report clinically meaningful decreases in pain, which has been defined for the BPI-SF as a 2-point difference in worst pain. ${ }^{83}$ Variability will be estimated using the mean/median scores and SD, in each group separately, at pretest and post-test. Similarly, sensitivity to change will be assessed by determining the number of participants who 
had a clinically meaningful increase in HRQoL scores over time. Although the study will not be powered to detect significant differences, we will use multiple regression to estimate the effect of group allocation on each outcome (separately) at post-test, adjusting for baseline scores. This will help determine the magnitude and direction of effect and provide a signal of the intervention's effectiveness. The analysis will be conducted using an intent-to-treat approach. As this is a pilot trial, no interim analyses are planned.

\section{PATIENT AND PUBLIC INVOLVEMENT}

Seven women (LC, CA, CF, DP, MP, BR, VSD) with cardiac pain formed the HEARTPA 9 N PAC. They were actively involved in phase 1 of this research programme (eg, defining search terms for our systematic review) and continue to be actively involved in setting the HEART$\mathrm{PA}+\mathrm{N}$ research agenda for phases $2 A, 2 B$ and 3. This includes assisting to define the scope of the project (eg, defining patient-reported outcome measures for the pilot RCT), active involvement in recruitment activities, assisting to write project quarterly newsletters and participation in all team meetings. They will be invited to be copresenters at scientific conference meetings and public forums (Café Scientifique) and will assist to write lay summaries and fact sheets for each phase of our project.

\section{ETHICS AND DISSEMINATION}

Ethics approval was obtained from the University of Toronto (36415; 26 November 2018). This is a 3-year study, phase 2A recruitment began in March 2019. Informed consent will be obtained from participants (online supplementary material 1 ). To ensure privacy during the pilot RCT, all personally identifying information will be stored on a separate database from health data on the app. Information that is sent to the smartphone or used by the reporting system will be independent of their personal information. No personal information will be transmitted after the initial set-up. For security issues, information that is transmitted will be sent securely via encrypted Hypertext Transfer Protocol Secure connection, preventing interception by a third party. All electronic entries will be backed up on a central server and communication with the central database server will occur through secure internet connections. Only the principal investigator and project coordinator will have access to the data. We will disseminate knowledge of HEARTPA우 $\mathrm{N}$ through publication, conference presentation and educational national public forums (Café Scientifiques), and through fact sheets, tweets and webinars posted in the Women's Xchange Knowledge Translation and Exchange Centre as well as to key stakeholders and programmes.

\section{Author affiliations}

${ }^{1}$ University of Toronto Lawrence S Bloomberg Faculty of Nursing, Toronto, Ontario,

Canada

${ }^{2}$ Pain Research Unit, University Health Network, Toronto, Ontario, Canada
${ }^{3}$ University of Toronto, Toronto, Ontario, Canada

${ }^{4}$ Department of Nursing and Health Promotion, Oslo Metropolitan University, Oslo, Norway

${ }^{5}$ Healthcare Human Factors, University Health Network, Toronto, Ontario, Canada

${ }^{6}$ Patient Advisor, Toronto, Ontario, Canada

${ }^{7}$ Women's College Hospital, Toronto, Ontario, Canada

${ }^{8}$ Faculty of Health - Department of Psychology, York University, Toronto, Ontario,

Canada

${ }^{9}$ The Peter Gilgan Centre for Research and Learning, Toronto, Ontario, Canada

${ }^{10}$ Institute of Nursing, Oslo Metropolitan University, Oslo, Akershus, Norway

${ }^{11}$ Médecine Familiale, Université Laval, Quebec, Quebec, Canada

${ }^{12}$ College of Nursing, Florida State University, Tallahassee, Florida, USA

${ }^{13}$ Faculty of Health Sciences, McMaster University, Hamilton, Ontario, Canada

${ }^{14}$ Faculty of Nursing, University of Alberta, Edmonton, Alberta, Canada

${ }^{15}$ Anishnawbe Health, Toronto, Ontario, Canada

${ }^{16}$ Medicine, McGill University, Montreal, Quebec, Canada

${ }^{17}$ Wasser Pain Management Centre, Sinai Health System, Toronto, Ontario, Canada

${ }^{18}$ Lawrence S Bloomberg Faculty of Nursing, University of Toronto, Toronto, Ontario, Canada

${ }^{19}$ Patient Advisor, Moose Factory, Ontario, Canada

Twitter Monica Parry @parryresearch, Michael McGillion @cardiacpainRC and Christine Faubert @Christine Faubert

Acknowledgements We thank the PAC for their active involvement in setting the HEARTPA $\mathrm{N}$ research agenda, including providing letters of support and defining patient-oriented outcome measures (PROM) for the pilot RCT. We also thank the Heart and Stroke Foundation for supporting HEARTPA $P$ N and working together to share the findings and the products generated from our work. The Women's Xchange will provide ongoing sex and gender consultative support throughout the project, opportunities for trainees to learn more about sex and gender integration in health research, and also assist with knowledge translation and exchange.

Contributors The PI (MP) and co-PI (HC) conceived the study. Co-PIs (AKB, JAC, LC, AD, PH, JK, CL, ML, FL, JMD, MM, CN, RP, LP, LPink, JP, JS, JCV, JWW, CA, $\mathrm{CF}, \mathrm{DP}, \mathrm{MP}, \mathrm{BR}, \mathrm{VSD}$ ) contributed to the study design and are assisting with study implementation across phases $2 \mathrm{~A}, 2 \mathrm{~B}$ and 3 . All authors are grant holders except our Human Factors Designers: ML, LP and AU. Our Human Factors Designers (ML, $\mathrm{LP}$ and $\mathrm{AU}$ ) are deeply involved in phases $2 \mathrm{~A}$ and $2 \mathrm{~B}$ (HEARTPA $\mathrm{N}$ content and core feature set/usability testing). JAC, CL and JS provided methodological expertise on mobile device functionality and the sequential phased approach to developing the HEARTPA $P$ N application. ML, AKB and JCV provided methodological expertise: ML and AKB will lead all qualitative analyses and JCV will lead the primary statistical analysis of the pilot RCT. Seven women (LC, CA, CF, DP, MP, BR, VSD) with cardiac pain formed the HEARTPA $P$ N PAC and are co-PIs. PH, FL, CN, JP and LP will inform and assist to validate our triage algorithms. All other authors will assist to build and/ or approve content for the HEARTPA $\mathrm{N}$ application (MP, HC, AD, JK, JMD, MM, RP, LPink, JWW). All authors approved the final manuscript prior to submission and are accountable for all aspects in ensuring accuracy and integrity of work across all phases of the study.

Funding This work was supported by a Canadian Institutes of Health Research (ClHR; 389044) Project Grant Fall 2017 Competition, Ottawa, Ontario, Canada.

Competing interests None declared.

Patient consent for publication Not required.

Ethics approval Ethics approval was obtained from the University of Toronto (36415) (26 November 2018).

Provenance and peer review Not commissioned; externally peer reviewed.

Open access This is an open access article distributed in accordance with the Creative Commons Attribution Non Commercial (CC BY-NC 4.0) license, which permits others to distribute, remix, adapt, build upon this work noncommercially, and license their derivative works on different terms, provided the original work is properly cited, appropriate credit is given, any changes made indicated, and the use is non-commercial. See: http://creativecommons.org/ licenses/by-nc/4.0/.

ORCID iDs

Monica Parry http://orcid.org/0000-0002-6941-1380

Ann Kristin Bjørnnes http://orcid.org/0000-0002-5356-3873 


\section{REFERENCES}

1 Johnston N, Schenck-Gustafsson K, Lagerqvist B. Are we using cardiovascular medications and coronary angiography appropriately in men and women with chest pain? Eur Heart $J$ 2011;32:1331-6.

2 Qureshi W, Blaha MJ, Nasir K, et al. Gender differences in coronary plaque composition and burden detected in symptomatic patients referred for coronary computed tomographic angiography. Int $J$ Cardiovasc Imaging 2013;29:463-9.

3 Kok MM, van der Heijden LC, Sen $\mathrm{H}$, et al. Sex difference in chest pain after implantation of newer generation coronary drug-eluting stents: a patient-level pooled analysis from the TWENTE and Dutch Peers trials. JACC Cardiovasc Interv 2016;9:553-61.

4 Parry M, Watt-Watson J, Hodnett E, et al. Pain experiences of men and women after coronary artery bypass graft surgery. $J$ Cardiovasc Nurs 2010;25:E9-15

5 King KM, Parry M, Southern D, et al. Women's recovery from Sternotomy-Extension (WREST-E) study: examining long-term pain and discomfort following sternotomy and their predictors. Heart 2008;94:493-7.

6 Bjørnnes AK, Parry M, Lie I, et al. Pain experiences of men and women after cardiac surgery. J Clin Nurs 2016;25:3058-68.

7 Choinière M, Watt-Watson J, Victor JC, et al. Prevalence of and risk factors for persistent postoperative nonanginal pain after cardiac surgery: a 2-year prospective multicentre study. CMAJ 2014;186:E213-23.

8 Bordoni B, Marelli F, Morabito B, et al. Post-sternotomy pain syndrome following cardiac surgery: case report. J Pain Res 2017;10:1163-9.

9 Agrawal S, Mehta PK, Bairey Merz CN. Cardiac syndrome X: update 2014. Cardiol Clin 2014;32:463-78.

10 Tamis-Holland JE, Jneid H, Reynolds HR, et al. Contemporary diagnosis and management of patients with myocardial infarction in the absence of obstructive coronary artery disease: a scientific statement from the American heart association. Circulation 2019;139:e891-908.

11 Thygesen K, Alpert JS, Jaffe AS, et al. Fourth universal definition of myocardial infarction (2018). J Am Coll Cardiol 2018;72:2231-64.

12 Wei J, Cheng S, Merz CNB. Coronary microvascular dysfunction causing cardiac ischemia in women. JAMA 2019;322:2334-5.

13 Pepine CJ, Ferdinand KC, Shaw LJ, et al. Emergence of nonobstructive coronary artery disease: a woman's problem and need for change in definition on angiography. J Am Coll Cardiol 2015;66:1918-33.

14 CDC. Health, United States, 2011 With a Special Feature on Socioeconomic Status and Health. National Center for Health Statistics, 2011.

15 Ferry AV, Anand A, Strachan FE, et al. Presenting symptoms in men and women diagnosed with myocardial infarction using sex-specific criteria. J Am Heart Assoc 2019;8:e012307.

16 Mehta LS, Beckie TM, DeVon HA, et al. Acute myocardial infarction in women: a scientific statement from the American heart association. Circulation 2016;133:1-32.

17 Canto JG, Canto EA, Goldberg RJ. Time to standardize and broaden the criteria of acute coronary syndrome symptom presentations in women. Can J Cardiol 2014;30:721-8.

18 Kirchberger I, Heier M, Wende R, et al. The patient's interpretation of myocardial infarction symptoms and its role in the decision process to seek treatment: the MONICA/KORA myocardial infarction registry. Clin Res Cardiol 2012;101:909-16.

19 Sjöström-Strand A, Fridlund B. Women's descriptions of symptoms and delay reasons in seeking medical care at the time of a first myocardial infarction: a qualitative study. Int J Nurs Stud 2008;45:1003-10.

20 von Eisenhart Rothe AF, Albarqouni L, Gärtner C, et al. Sex specific impact of prodromal chest pain on pre-hospital delay time during an acute myocardial infarction: findings from the multicenter Medea study with 619 STEMI patients. Int J Cardiol 2015;201:581-6.

21 Sabbag A, Matetzky S, Gottlieb S, et al. Recent temporal trends in the presentation, management, and outcome of women hospitalized with acute coronary syndromes. Am J Med 2015;128:380-8.

22 Arthur HM, Campbell P, Harvey PJ, et al. Women, cardiac syndrome $X$, and microvascular heart disease. Can J Cardiol 2012;28:S42-9.

23 Altintas E, Yigit F, Taskintuna N. The impact of psychiatric disorders with cardiac syndrome $X$ on quality of life: 3 months prospective study. Int J Clin Exp Med 2014;7:3520-7.

24 Pacheco C, Bairey Merz CN. Women in cardiovascular clinical Trials-What are the barriers to address to improve enrollment? Can J Cardiol 2019;35:552-4.

25 Kuehn BM. Boosting women's participation in cardiovascular trials. Circulation 2018;138:1366-7.
26 Parry M, Bjørnnes AK, Clarke H, et al. Self-Management of cardiac pain in women: an evidence MAP. BMJ Open 2017;7:e018549.

27 Lorig KR, Holman H. Self-Management education: history, definition, outcomes, and mechanisms. Ann Behav Med 2003;26:1-7

28 Bandura A. Self-Efficacy: toward a unifying theory of behavioral change. Psychol Rev 1977;84:191-215.

29 Johnston M, Foster M, Shennan J, et al. The effectiveness of an acceptance and commitment therapy self-help intervention for chronic pain. Clin J Pain 2010;26:393-402.

30 McGillion M, Arthur H, Victor JC, et al. Effectiveness of psychoeducational interventions for improving symptoms, healthrelated quality of life, and psychological well being in patients with stable angina. Curr Cardiol Rev 2008;4:1-11.

31 Perry KN, Nicholas MK, Middleton J. Multidisciplinary cognitive behavioural pain management programmes for people with a spinal cord injury: design and implementation. Disabil Rehabil 2011;33:1272-80.

32 Naylor MR, Keefe FJ, Brigidi B, et al. Therapeutic interactive voice response for chronic pain reduction and relapse prevention. Pain 2008;134:335-45.

33 Dysvik E, Kvaløy JT, Stokkeland R, et al. The effectiveness of a multidisciplinary pain management programme managing chronic pain on pain perceptions, health-related quality of life and stages of change--A non-randomized controlled study. Int J Nurs Stud 2010;47:826-35.

34 Glombiewski JA, Hartwich-Tersek J, Rief W. Two psychological interventions are effective in severely disabled, chronic back pain patients: a randomised controlled trial. Int J Behav Med 2010;17:97-107.

35 Parry M, Bjørnnes AK, Victor JC, et al. Self-Management interventions for women with cardiac pain: a systematic review and meta-analysis. Can J Cardiol 2018;34:458-67.

36 Knight-Agarwal C, Davis DL, Williams L, et al. Development and pilot testing of the eating 4two mobile phone APP to monitor gestational weight gain. JMIR Mhealth Uhealth 2015;3:e44.

37 Willcox JC, Campbell KJ, McCarthy EA, et al. Testing the feasibility of a mobile technology intervention promoting healthy gestational weight gain in pregnant women (txt4two) - study protocol for a randomised controlled trial. Trials 2015;16:1-8.

38 Waring ME, Moore Simas TA, Xiao RS, et al. Pregnant women's interest in a website or mobile application for healthy gestational weight gain. Sex Reprod Healthc 2014;5:182-4.

39 Fukuoka Y, Komatsu J, Suarez L, et al. The mPED randomized controlled clinical trial: applying mobile persuasive technologies to increase physical activity in sedentary women protocol. BMC Public Health 2011:11:1-8.

40 Thomas JG, Wing RR. Health-e-call, a smartphone-assisted behavioral obesity treatment: pilot study. JMIR Mhealth Uhealth 2013;1:e3.

41 Llanos AAM, Krok JL, Peng J, et al. Effects of a walking intervention using mobile technology and interactive voice response on serum adipokines among postmenopausal women at increased breast cancer risk. Horm Cancer 2014;5:98-103.

42 Wen K-Y, Miller SM, Kilby L, et al. Preventing postpartum smoking relapse among inner City women: development of a theory-based and evidence-guided text messaging intervention. JMIR Res Protoc 2014;3:e20

43 Licskai C, Sands TW, Ferrone M. Development and pilot testing of a mobile health solution for asthma self-management: asthma action plan smartphone application pilot study. Can Respir $J$ 2013;20:301-6.

44 CATALYST. Smartphone behaviour in Canada and the implications for marketers in 2016, 2016. Available: http://catalyst.ca/2016-canadiansmartphone-behaviour/

45 Life in the fast lane: how are Canadians managing? 2017. Available: https://www150.statcan.gc.ca/n1/daily-quotidien/171114/ dq171114a-eng.htm?HPA=1 [Accessed 20 Dec 2019].

46 Silow-Carroll S, Smith B. Clinical management apps: creating partnerships between providers and patients. Issue Brief 2013;30:1-10.

47 Fawcett J, Watson J, Neuman B, et al. On nursing theories and evidence. J Nurs Scholarsh 2001;33:115-9.

48 Ryan P, Sawin KJ. The individual and family self-management theory: background and perspectives on context, process, and outcomes. Nurs Outlook 2009;57:217-25.

49 Danaher BG, Brendryen H, Seeley JR, et al. From black box to toolbox: Outlining device functionality, engagement activities, and the pervasive information architecture of mHealth interventions. Internet Interventions 2015;2:91-101. 
50 Craig P, Dieppe P, Macintyre S, et al. Developing and evaluating complex interventions: the new medical Research Council guidance. BMJ 2008;337:1-6.

51 MRC. A framework for development and evaluation of RCTs fro complex interventions to improve health 2000.

52 Campbell M, Fitzpatrick R, Haines A, et al. Framework for design and evaluation of complex interventions to improve health. BMJ 2000;321:694-6.

53 Danaher BG, Seeley JR. Methodological issues in research on webbased behavioral interventions. Ann Behav Med 2009;38:28-39.

54 Holzinger A. Usability engineering methods for software developers. Commun ACM 2005;48:71-4

55 Callahan CM, Unverzagt FW, Hui SL, et al. Six-item screener to identify cognitive impairment among potential subjects for clinical research. Med Care 2002;40:771-81.

56 Carpenter CR, DesPain B, Keeling TN, et al. The Six-Item Screener and AD8 for the detection of cognitive impairment in geriatric emergency department patients. Ann Emerg Med 2011;57:653-61.

57 Kvale S, Brinkmann S. Interviews: Learning the craft of qualitative interviewing. Los Angeles: Sage, 2015.

58 Dicicco-Bloom B, Crabtree BF. The qualitative research interview. Med Educ 2006;40:314-21

59 Jibb LA, Stevens BJ, Nathan PC, et al. A smartphone-based pain management APP for adolescents with cancer: establishing system requirements and a pain care algorithm based on literature review, interviews, and consensus. JMIR Res Protoc 2014;3:e15.

60 Stinson J, Gupta A, Dupuis F, et al. Usability testing of an online selfmanagement program for adolescents with cancer. $J$ Pediatr Oncol Nurs 2015;32:70-82.

61 Breakey VR, Warias AV, Ignas DM, et al. The value of usability testing for Internet-based adolescent self-management interventions: "Managing Hemophilia Online". BMC Med Inform Decis Mak 2013;13:113.

62 Macefield R. How to specify the participant group size for usabiltiy studies: a practitioner's guide. Journal of Usability Studies 2009;5:34-45.

63 Nielsen J, Landauer T. A mathematical model of the finding of usability problems. Paper presented at: Proceedings of ACM INTERCHI'93 Conference; 24-29 April 1993, Amsterdam, The Netherlands, 1993.

64 Jaspers MWM. A comparison of usability methods for testing interactive health technologies: methodological aspects and empirical evidence. Int J Med Inform 2009;78:340-53.

65 Brooke J. A "quick and dirty" usabilty scale. In: Jordan P, Thomas B, Weerdmeester B, et al, eds. Usability evaluation in industry. London: Taylor \& Francis, 1996.

66 Bangor A, Kortum PT, Miller JT. An empirical evaluation of the system usability scale. Int J Hum Comput Interact 2008;24:574-94.
67 Kushniruk A. Evaluation in the design of health information systems: application of approaches emerging from usability engineering. Comput Biol Med 2002;32:141-9.

68 Biørn-Hansen A, Majchrzak T, Grønli T. Progressive web Apps: the possible web-native unifier for mobile development. Proceedings of the 13th International Conference on Web Information Systems and Technologies WEBIST, 2017.

69 Majchrzak T, Biørn-Hansen A, Grønli T. Progressive web Apps: the definite approach to Cross-Platform development. Proceedings of the 51st Hawaii International Conference on System Sciences, 2018.

70 Malavolta I. Beyond native apps: web technologies to the rescue! Paper presented at: Mobile! '162016; Amsterdam, Netherlands.

71 Cotugna N, Vickery CE, Carpenter-Haefele KM. Evaluation of literacy level of patient education Pages in health-related journals. $J$ Community Health 2005;30:213-9.

72 Parry MJ, Watt-Watson J, Hodnett E, et al. Cardiac home education and support trial (chest): a pilot study. Can J Cardiol 2009;25:e393-8.

73 Suresh K. An overview of randomization techniques: an unbiased assessment of outcome in clinical research. $J$ Hum Reprod Sci 2011:4:8-11.

74 Kernan WN, Viscoli CM, Makuch RW, et al. Stratified randomization for clinical trials. J Clin Epidemiol 1999;52:19-26.

75 Tariman JD, Berry DL, Halpenny B, et al. Validation and testing of the acceptability E-scale for web-based patient-reported outcomes in cancer care. Appl Nurs Res 2011;24:53-8.

76 McDowell I, Newell C. Measuring health: A guide to rating scales and questionnaires.2 edn. New York: Oxford University Press, 1996.

77 Watt-Watson J, Stevens B, Katz J, et al. Impact of preoperative education on pain outcomes after coronary artery bypass graft surgery. Pain 2004;109:73-85.

78 Watt-Watson J, Stevens B, Costello J, et al. Impact of preoperative education on pain management outcomes after coronary artery bypass graft surgery: a pilot. Can J Nurs Res 2000;31:41-56.

79 Ware J, Kosinski M, Dewey J. How to score version 2 of the SF-36 health survey: a user's manual. in. Boston: The Health Institute, New England Medical Center, 2002.

80 Irvine D, O'Brien-Pallas LL, Murray M, et al. The reliability and validity of two health status measures for evaluating outcomes of home care nursing. Res Nurs Health 2000;23:43-54.

81 Ware J. Sf-36 health Survery: Manueal and interpretation guide. in. Boston: New England Medical Center, The Health Institute, 1993.

82 Thabane L, Ma J, Chu R, et al. A tutorial on pilot studies: the what, why and how. BMC Med Res Methodol 2010;10.

83 Mathias SD, Crosby RD, Qian Y, et al. Estimating minimally important differences for the worst pain rating of the brief pain Inventory-Short form. J Support Oncol 2011;9:72-8. 